\title{
One-Dimensional Cellular Automata with Reflective Boundary Conditions and Radius Three
}

\author{
H. $\operatorname{AKIN}^{a}$, I. SIAP ${ }^{b}$ AND S. UGUZ ${ }^{c}$ \\ ${ }^{a}$ Department of Mathematics, Education Faculty, Zirve University, 27260, Gaziantep, Turkey \\ ${ }^{b}$ Department of Mathematics, Faculty of Art and Sciences, Yildiz Technical University, 34210, Istanbul, Turkey \\ ${ }^{c}$ Department of Mathematics, Faculty of Art and Sciences, Harran University, 63000, Istanbul, Turkey \\ A family of one-dimensional finite linear cellular automata with reflective boundary condition over the field $\boldsymbol{Z}_{p}$ \\ is defined. The generalizations are the radius and the field that states take values. Here, we establish a connection \\ between reversibility of cellular automata and the rule matrix of the cellular automata with radius three. Also, we \\ prove that the reverse $\mathrm{CA}$ of this family again falls into this family.
}

DOI: 10.12693 /APhysPolA.125.405

PACS: 02.10.Yn, 07.05.Kf, 02.10.Ox

\section{Preliminaries}

In this section, we define $1 \mathrm{D}$ finite cellullar automata (CAs) with reflective boundary conditions (shortly RBC) over primitive fields with $p$ elements $\boldsymbol{Z}_{p}=$ $\{0,1, \ldots, p-1\}$, where $p \geq 2$ is prime. This definition is a natural generalization of particular $1 \mathrm{D}$ null boundary CAs. As a special case with $p=2$ the structure and reversibility problem over binary fields is studied by del Rey and Sanchez et al. in [1] and primitive fields is studied by Cinkir et al. in [2] and Siap et al. in [3], respectively. The approach of studying the algebraic structure and their reversibility property for this general case is generalized from [3]. For the binary field case, Koroglu et al. [4] have shown that $1 \mathrm{D}$ finite CAs with null boundary conditions based error correcting codes have faster decoding algorithm than the classical linear syndrome decoding algorithm. Depending on the neighborhood of the extreme cells, there exist three approaches:

The periodic boundary $C A[2,3]$,

The null boundary $C A[1,5]$,

The reflective boundary $C A[6]$.

In this paper, we will only deal with reflective boundary conditions. In [1], the reversibility problem for linear CA (LCA) with reflective boundary defined by a rule matrix in the form of a diagonal matrix has been studied over the binary field recently, this work has been extended mainly to ternary fields and partial answers regarding the reversibility are addressed also therein. A doubly-infinite sequence $x=\left(x_{n}\right)_{n=-\infty}^{\infty}$, where the entries are from $\boldsymbol{Z}_{p}$ where $p$ is a prime number is an element of the space $\boldsymbol{Z}_{p}^{Z}$. A CA is a map defined by $T_{f}: Z_{p}^{Z} \rightarrow Z_{p}^{Z}$ where $\left(T_{f} x\right)_{i}=f\left(x_{i-r}, \ldots, x_{i+r}\right)$, where $f: \boldsymbol{Z}_{p}^{2 r+1} \rightarrow \boldsymbol{Z}_{p}$ is called local rule. Martin et al. [7] showed that if a local rule $f$ is linear, i.e.,

$$
f\left(x_{-r}, \ldots, x_{r}\right)=\sum_{i=-r}^{r} a_{i} x_{i}(\bmod p),
$$

where at least one of $a_{-r}, \ldots, a_{r}$ is nonzero $\bmod p$, then $T_{f}$ is $1 \mathrm{D}$ linear CA. A 1D LCA $T_{f[-r, r]}$ is defined by the local rule $f$ given in Eq. (1.1):

$$
\begin{aligned}
& \left(T_{f}[-r, r] x\right)=\left(y_{n}\right)_{n=-\infty}^{\infty}, \\
& y_{n}=f\left(x_{n-r}, \ldots, x_{n+r}\right)=\sum_{i=-r}^{r} a_{i} x_{n+i}(\bmod p),
\end{aligned}
$$

where $a_{-r}, \ldots, a_{r} \in \boldsymbol{Z}_{p}$ (see [7] for details). In this paper, we will only consider the $1 \mathrm{D}$ finite LCA defined by local rule (1.2) under modulo $p$ algebra and we further assume that radius $r$ and $p \geq 2$ is a prime number. CAs can also be defined by special local rules; next state cell is determined by all neighboring cells within radius $r$ such that the first and last cell are assumed to be next to each other in a cyclic manner.

Let us consider the vector $C^{t}=$ $\left[x_{1}^{t}, x_{2}^{t}, x_{3}^{t}, \ldots, x_{n-1}^{t}, x_{n}^{t}\right] \in Z_{p}^{n}$. The vector $C^{t}$ is called a configuration of the $1 \mathrm{D}$ finite LCA at time $t$, therefore $C^{0}$ is the initial configuration. The configuration length of a vector will be assumed to be $n$ in this paper. Hence, it is clear that $n \geq 7$. Reflective boundary conditions are obtained by reflecting the lattice at the boundary. In this case, in order to obtain neighboring cell values for them the cells states at the extremities are repeated. In one dimension, reflective boundaries and their iterations under the $1 \mathrm{D}$ finite $\mathrm{CA} A_{n}$ can be arranged by

$$
\begin{aligned}
& \left.x_{3}^{t} x_{2}^{t} x_{1}^{t}\right]\left[x_{1}^{t} x_{2}^{t} \ldots x_{n-1}^{t} x_{n}^{t}\right]\left[x_{n}^{t} x_{n-1}^{t} x_{n-2}^{t} \stackrel{\mathrm{A}_{n}^{(r)}}{\longrightarrow}\right. \\
& \left.x_{3}^{t+1} x_{2}^{t+1} x_{1}^{t+1}\right]\left[x_{1}^{t+1} x_{2}^{t+1} \ldots x_{n-1}^{t+1} x_{n}^{t+1}\right]\left[x_{n}^{t+1} x_{n-1}^{t+1} x_{n-2}^{t+1} .\right.
\end{aligned}
$$

Let us define the $1 \mathrm{D}$ finite CA $A_{n}(n \geq 2 r+1)$ with RBC. Therefore, we have to define the local rule with RBC.

Proposition 1.1. The local rule under reflective boundary condition (1.3) is given by the following expression:

$$
\begin{aligned}
& x_{1}^{t+1}=\left(a_{0}+a_{-1}\right) x_{1}^{t}+\left(a_{1}+a_{-2}\right) x_{2}^{t} \\
& \quad+\left(a_{2}+a_{-3}\right) x_{3}^{t}+a_{3} x_{4}^{t} \bmod p, \\
& x_{2}^{t+1}=\left(a_{-1}+a_{-2}\right) x_{1}^{t}+\left(a_{0}+a_{-3}\right) x_{2}^{t} \\
& \quad+a_{1} x_{3}^{t}+a_{2} x_{4}^{t}+a_{3} x_{5}^{t} \bmod p, \\
& x_{3}^{t+1}=\left(a_{-3}+a_{-2}\right) x_{1}^{t}+\sum_{k=2}^{6} a_{k-3} x_{k}^{t} \bmod p,
\end{aligned}
$$




$$
x_{i>3}^{t+1} \equiv\left\{\begin{array}{l}
\sum_{k=0}^{6} a_{k-3} x_{k+1+i}^{t} \bmod p, \quad 4 \leq i \leq n-3 \\
\sum_{k=0}^{4} a_{k-3} x_{n+k-4}^{t}+\left(a_{2}+a_{3}\right) x_{n}^{t} \bmod p \\
i=n-2 \\
a_{-3} x_{n-4}^{t}+a_{-2} x_{n-3}^{t}+a_{-1} x_{n-2}^{t} \\
\quad+\left(a_{0}+a_{3}\right) x_{n-1}^{t}+\left(a_{1}+a_{2}\right) x_{n}^{t} \bmod p \\
i=n-1 \\
a_{-3} x_{n-3}^{t}+\left(a_{-2}+a_{3}\right) x_{n-2}^{t} \\
\quad+\left(a_{-1}+a_{2}\right) x_{n-1}^{t}+\left(a_{0}+a_{1}\right) x_{n}^{t} \bmod p \\
\quad i=n,
\end{array}\right.
$$

where $a_{i} \in Z_{p} \backslash\{0\}$ and $x_{i}^{t}$ is a symbol of the state of the $i$-th cell at time $t$. A common approach for defining a CA which merely depends on its local rule is to introduce a numbering label. By examining the relation among the states we see that the next cell $x_{i}^{t+1}$ state is obtained by the relation of the previous and the neighboring state of the cells within the radius $r$. So accordingly we introduce the following ordering and hence the numbering of the rules:

$$
\begin{aligned}
x_{i}^{t+1} & =\sum j=-r^{r} a_{j} x_{i+j}^{t} \\
\Rightarrow \mathrm{RN} & =\sum_{k=-r}^{r} a_{k} p^{r+k}=\left(a_{r} \ldots a_{1} a_{0} a_{-1} \ldots a_{-r}\right)_{p} .
\end{aligned}
$$

For instance, if $r=2, p=3$, and $\left(a_{-2}, a_{-1}, a_{0}, a_{1}, a_{2}\right)=$ $(2,2,1,1,1)$ then this rule will be named as rule number (RN) $3^{4}+3^{3}+3^{2}+2 \cdot 3^{1}+2 \cdot 3^{0}=(11122)_{3}=125$ or if $r=1, p=5$, and $\left(a_{-1}, a_{0}, a_{1}\right)=(2,1,1)$ then this rule will be named as rule number (RN) $5^{2}+5^{1}+2 \cdot 5^{0}=$ $(112)_{5}=32$. This presentation not only identifies the rules uniquely but it is also practical. Further, since each cell is assumed to affect the transitions we always assume that $a_{i} \in Z_{p} \backslash\{0\}$.

Theorem 1.2. The rule matrix $M_{n}^{(3)}$ corresponding to an $1 \mathrm{D}$ finite $\mathrm{CA} A_{n}^{(3)}$ generated by the local rule (1.4) with $\mathrm{RBC}$ is given by

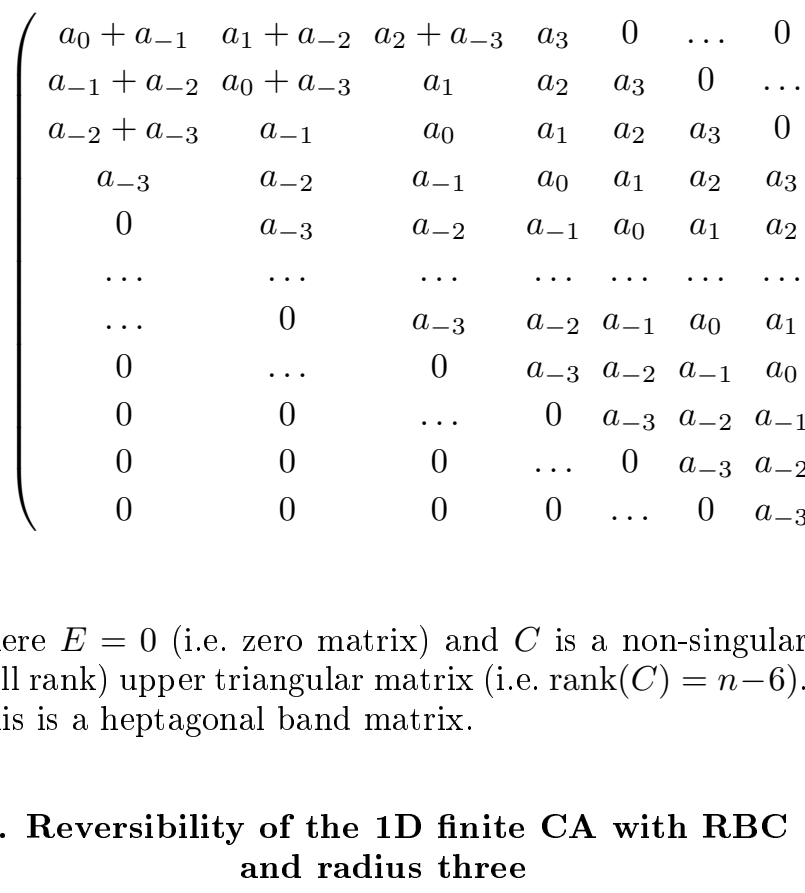

In this section, we study the inverse of the rule matrix $M_{n}^{(3)}$ corresponding to the 1D finite LCA $A_{n}$. To obtain the inverse of the matrix (1.6), we will compute the rank of the matrix (1.6). Our strategy to find the rank of $M_{n}$ is as follows: first we consider $M_{n}$ as

$$
M_{n}^{(3)}=\left(\begin{array}{cc}
A & B \\
C & D \\
E & F
\end{array}\right) \sim\left(\begin{array}{cc}
A & B \\
E & F \\
C & D
\end{array}\right) \sim\left(\begin{array}{cc}
0 & \bar{B} \\
0 & \bar{F} \\
C & D
\end{array}\right),
$$

where the symbol $\sim$ shows row-column operations of the matrix $M_{n}^{(3)}$, and $\bar{B}$ and $\bar{F}$ are matrices obtained from a suitable row-column operations. It can be seen from 2.1 that the rank of $M_{n}^{(3)}$ is

$\left.\begin{array}{ccc}0 & 0 & 0 \\ 0 & 0 & 0 \\ \ldots & 0 & 0 \\ 0 & \cdots & 0 \\ a_{3} & 0 & \ldots \\ \ldots & \ldots & \cdots \\ a_{2} & a_{3} & 0 \\ a_{1} & a_{2} & a_{3} \\ a_{0} & a_{1} & a_{2}+a_{3} \\ a_{-1} & a_{0}+a_{3} & a_{1}+a_{2} \\ a_{-2}+a_{3} & a_{-1}+a_{2} & a_{0}+a_{1}\end{array}\right)=\left(\begin{array}{cc}A & B \\ C & D \\ E & F\end{array}\right)$,

$$
\begin{aligned}
& \operatorname{rank}\left(M_{n}\right)=\operatorname{rank}(C)+\operatorname{rank}\left(\begin{array}{c}
\bar{B} \\
\bar{F}
\end{array}\right) \\
& =(n-6)+\operatorname{rank}\left(\begin{array}{c}
\bar{B} \\
\bar{F}
\end{array}\right) .
\end{aligned}
$$

So the problem of finding the rank reduces dramatically. Now let us compute this relation explicitly.

\subsection{A computational method for the rank of $M_{n}^{(3)}$}

Let $T_{i}$ denote the $i$-th row and $T_{i}[j]$ denote the $j$ the entry of the $i$-th row of matrix $T$, respectively. By definition, we have

$$
\begin{aligned}
T_{1}= & {\left[a_{0}+a_{-1}, a_{1}+a_{-2}, a_{2}+a_{-3}, a_{3}, 0, \ldots 0,0,0,0\right] } \\
T_{2}= & {\left[a_{-1}+a_{-2}, a_{0}+a_{-3}, a_{1}, a_{2}, a_{3}, 0, \ldots, 0\right] } \\
T_{3}= & {\left[a_{-2}+a_{-3}, a_{-1}, a_{0}, a_{1}, a_{2}, a_{3}, 0,0, \ldots, 0\right] } \\
T_{4}= & {\left[a_{-3}, a_{-2}, a_{-1}, a_{0}, a_{1}, a_{2}, a_{3}, 0,0,0, \ldots, 0\right] } \\
& \quad \ldots \\
T_{n-2}= & {\left[0,0,0, \ldots, 0,0,0, a_{-3}, a_{-2}, a_{-1}, a_{0}, a_{1},\right.} \\
a_{2} & \left.+a_{3}\right]
\end{aligned}
$$




$$
\begin{aligned}
& T_{n-1}=\left[0,0, \ldots, 0,0, a_{-3}, a_{-2}, a_{-1}, a_{0}+a_{3}, a_{1}+a_{2}\right] \\
& T_{n}=\left[0,0, \ldots, 0,0, a_{-3}, a_{-2}+a_{3}, a_{-1}+a_{2}, a_{0}+a_{1}\right] \\
& T_{i}=[\ldots] \in M_{1 \times n}\left(\boldsymbol{Z}_{p}\right) \text { for } i=1 \text { to } n .
\end{aligned}
$$

Define the map $\sigma$ as follows: $\sigma: M_{1 \times n}\left(\boldsymbol{Z}_{p}\right) \rightarrow$ $M_{1 \times n}\left(\boldsymbol{Z}_{p}\right), \quad \sigma\left(\left[x_{1}, x_{2}, x_{3}, \ldots, x_{n-1}, x_{n}\right]\right)=\left[0, x_{1}, x_{2}\right.$, $\left.x_{3}, \ldots, x_{n-1}\right]$. Also, if $X=\left[x_{1}, x_{2}, x_{3}, \ldots, x_{n-1}, x_{n}\right]$, then $X[i]=x_{i}$ represents the $i$-th entry of $X$. Further $y\left[x_{1}, x_{2}, \ldots, x_{n}\right]=\left[y x_{1}, y x_{2}, \ldots, y x_{n}\right]$. The following theorem presents an algorithm for computing the rank of $M_{n}^{(3)}$.

Theorem 2.1. Let the matrix $M_{n}^{(3)}$ with $n \geq 7$ represents the one-dimensional cellular automata over $\boldsymbol{Z}_{p}$ fields. Let

$$
T_{i}^{(1)}=T_{i}, \quad T_{i}^{(k+1)}=-\frac{1}{a_{-3}} T_{i}^{(k)}[k] \sigma^{(k-1)}\left(T_{4}\right)+T_{i}^{(k)}
$$

for $i=1$ to $n$ and $k=1$ to $n-6$.

Define the following $6 \times(n-6)$ block matrix consisting of blocks of square matrices of order $n$.

$B=$

$\left(\begin{array}{ccccc}T_{1}^{(n-6)}[n-6] & T_{1}^{(n-6)}[n-5] & \ldots & T_{1}^{(n-6)}[n-1] & T_{1}^{(n-1)}[n] \\ T_{2}^{(n-6)}[n-6] & T_{2}^{(n-6)}[n-5] & \ldots & T_{2}^{(n-6)}[n-1] & T_{2}^{(n-1)}[n] \\ T_{3}^{(n-6)}[n-6] & T_{3}^{(n-6)}[n-5] & \ldots & T_{3}^{(n-6)}[n-1] & T_{3}^{(n-1)}[n] \\ T_{n-2}^{(n-6)}[n-6] & T_{n-2}^{(n-6)}[n-5] & \ldots & T_{n-2}^{(n-6)}[n-1] & T_{n-2}^{(n-6)}[n] \\ T_{n-1}^{(n-6)}[n-6] & T_{n-1}^{(n-6)}[n-5] & \ldots & T_{n-1}^{(n-6)}[n-1] & T_{n-1}^{(n-6)}[n] \\ T_{n}^{(n-6)}[n-6] & T_{n}^{(n-6)}[n-5] & \ldots & T_{n}^{(n-6)}[n-1] & T_{1}^{(n-6)}[n]\end{array}\right)$

Then, $\operatorname{rank}\left(M_{n}^{(3)}\right)=(n-6)+\operatorname{rank}(B)$. A straightforward corollary which gives a lower bound for the rank of a cellular automaton is presented below.

Corollary. Let $M_{n}^{(3)}$ be the rule matrix corresponding to a cellular automaton defined above. Then, $(n-6) \leq$ $\operatorname{rank}\left(M_{n}^{(3)}\right) \leq n$.

\subsection{An algorithm for computing the rank of $M_{n}^{(3)}$}

Now we can summarize the method introduced above for computing the rank of $M_{n}^{(3)}$ as follows:

Step 1. Determine the first three rows $T_{1}, T_{2}, T_{3}$ and the last three rows $T_{n-2}, T_{n-1}, T_{n}$ of $M_{n}$ which consists of block of matrices. Set $T_{1}^{(1)}=T_{1}, T_{2}^{(1)}=T_{2}, T_{3}^{(1)}=T_{3}$, and $T_{n-2}^{(1)}=T_{n-2}, T_{n-1}^{(1)}=T_{n-1}, T_{n}^{(1)}=T_{n}$.

Step 2. For $1 \leq k \leq n-1$, compute $T_{1}^{(k+1)}=-\frac{1}{a_{-3}} T_{1}^{(k)}[k] \sigma^{(k-1)}\left(T_{4}\right)+T_{1}^{(k)}, T_{2}^{(k+1)}=$ $-\frac{1}{a_{-3}} T_{2}^{(k)}[k] \sigma^{(k-1)}\left(T_{4}\right)+T_{2}^{(k)}$ and the other $T_{i}^{(k)}$ for $i=3, n-2, n-1, n$.
Hence, determining the matrix $B$ for every iterations, compute $T_{i}^{(k)}(i=1,2,3, n-2, n-1, n)$, since $B$ is a matrix, arithmetics can be carried out modulo $p$ (prime number $p$ ) the characteristic polynomial of $B$ which saves reasonable time.

Step 3. Compute the rank of $B$. Therefore, $\operatorname{rank}\left(M_{n}^{(3)}\right)=(n-6)+\operatorname{rank}(B)$.

\section{Conclusion}

In this paper we studied the reversibility problem of the family of $1 \mathrm{D}$ finite CAs with reflective boundary. The reversibility problem becomes easy to solve computing the rank of the matrix. If the rule matrix corresponding to the $1 \mathrm{D}$ finite linear $\mathrm{CA}$ has full rank, then the $\mathrm{CA}$ is reversible, otherwise the $\mathrm{CA}$ is irreversible. Some other interesting results and further connections on this direction wait to be explored in 2D CA's [8-10].

\section{Acknowledgments}

This work is supported by the Scientific and Technological Research Council of Turkey (TÜBİTAK) (project number: 110T713).

\section{References}

[1] A. Martin del Rey, G. Rodriguez Sánchez, Appl. Math. Comput. 217, 8360 (2011).

[2] Z. Cinkir, H. Akin, I. Siap, J. Stat. Phys. 143, 807 (2011).

[3] I. Siap, H. Akin, M.E. Koroglu, Int. J. Mod. Phys. C 23, 1 (2012).

[4] M.E. Koroglu, I. Siap, H. Akin, Arab. J. Sci. Eng., 2013, accepted for publication.

[5] L. Hernández Encinas, A. Martin del Rey, Appl. Math. Comput. 189, 1782 (2007).

[6] H. Akin, F. Sah, I. Siap, Int. J. Mod. Phys. C 23, 1 (2012).

[7] O. Martin, A.M. Odlyzko, S. Wolfram, Commun Math. Phys. 93, 219 (1984).

[8] I. Siap, H. Akin, S. Uguz, Comput. Math. Appl. 62, 4161 (2011)

[9] S. Uguz, H. Akin, I. Siap, Int. J. Bifurcat. Chaos. 23, 1350101 (2013).

[10] S. Uguz, U. Sahin, H. Akin, I. Siap, Int. J. Bifurcat. Chaos. 24, 25 (2014). 